\title{
Selected Topics on Business Informatics: Editorial Introduction to Issue 19 of CSIMQ
}

\author{
Kurt Sandkuhl $^{1 *}$ and Jelena Zdravkovic ${ }^{2}$ \\ ${ }^{1}$ University of Rostock, Albert-Einstein-Str. 22, 18059 Rostock, Germany \\ 2 Stockholm University, Box 7003, Kista, 16407, Sweden \\ kurt.sandkuhl@uni-rostock.de, jelenaz@dsv.su.se
}

Business Informatics is the scientific discipline combining computer science, business administration and information technology and investigating related phenomena in their socio-economical context, including companies, organizations, administrations, and society in general. As a field of study, it endeavors to take a systematic and analytic approach in adopting a multi-disciplinary orientation that draws theories and practices from the fields of management science, organizational science, computer science, systems engineering, information systems, information management, social science, and economics information science.

The objective of this thematic issue was twofold. The first it was to present selected research results of the 17th International Conference on Perspectives in Business Informatics Research (BIR2018) in Stockholm, Sweden, in September 24-26, 2018. The conference created a productive forum for researchers and practitioners on the specific topic - Business Resilience, with the intention of exploring organizational and information system resilience in congruence. Top papers were selected by the Program Chairs Prof. Janis Grabis and Prof. Jelena Zdravkovic to submit extended versions for a possible publication to this thematic issue. The second objective was to consider external candidate submissions for bringing attention to recent research in the Business Informatics discipline and thus make this issue even more actual.

The thematic issue collection opens with the article entitled "Ecosystem Architecture Management in the Public Sector - From Problems to Solutions", which, by taking the focus concerning enterprise architecture (EA) to the Finnish public sector, discusses how EA concept and tool need to be developed to support government business ecosystem and organization design. The research results indicate that, beyond federal or regional government, even a single municipality can be perceived as an ecosystem of its sectoral domains, subsidiaries and such. Based on the data from smart city cases in Finland, the authors suggest a management model for the governmental ecosystem architecture design, specifically a design process for co-creating new services in the ecosystem.

The second article, "A Modeling Approach for Getting to Win-Win in Industrial

\footnotetext{
* Corresponding author
}

(C) 2019 Kurt Sandkuhl et al. This is an open access article licensed under the Creative Commons Attribution License (http://creativecommons.org/licenses/by/4.0).

Reference: K. Sandkuhl and J. Zdravkovic, "Selected Topics on Business Informatics: Editorial Introduction to Issue 19 of CSIMQ," Complex Systems Informatics and Modeling Quarterly, CSIMQ, no. 19, pp. I-II, 2019. Available: https://doi.org/10.7250/csimq.2019-19.00

Additional information. Author ORCID iD: K. Sandkuhl - https://orcid.org/0000-0002-7431-8412, J. Zdravkovic https://orcid.org/0000-0002-0870-0330. PII S225599221900108X. Received: 30 June 2019. Available online: 19 July 2019. 
Collaboration under Strategic Coopetition" addresses a relationship in which two or more organizations cooperate and compete simultaneously. Actors under coopetition cooperate to achieve collective objectives and compete to maximize their individual benefits. The study proposes a strategic modeling approach that involves goal-modeling to explore strategic alternatives of actors with Game Tree decision-modeling to evaluate the actions and payoffs. It demonstrates the activation of the approach for systematically searching for alternatives to generate a win-win strategy. An illustrative industrial scenario on the Industrial Data Space, a platform helping organizations to overcome obstacles to data sharing in a coopetitive ecosystem, is used to demonstrate the research contribution.

"Automated Modeling with Abstraction for Enterprise Architecture (AMA4EA): Business Process Model Automation in an Industry 4.0 Laboratory" is the third article aiming to examine the transformation towards the Industry 4.0, with the concern of managing large amounts of data to extract value from them. To solve this challenge, the study proposes the use an Enterprise Architecture method to support companies to automatically abstract data from enterprise systems to concepts, and then to automatically create an EA model. The method is demonstrated at an Industry 4.0 laboratory showing how the data of business processes from the Enterprise Resource Planning (ERP) system and Manufacturing Execution System (MES) could be extracted, documented and visualized.

The fourth article "Discussing Hierarchic Viewpoints Theoretical Considerations and a Practical Example" analyzes Governance, Risk and Compliance (GRC) systems as vital to managing risks threatening modern enterprises. The key constituent to GRC systems is the definition of Controls implemented as a concern on the different layers of an Enterprise Architecture (EA), allowing to use an EA viewpoint to cover Control compliance assessments. The study explores and deepens this relationship, and derives a metamodel linking Control and EA. The research results are complemented with an instantiation of Control compliance applied in a global enterprise in the insurance industry.

The Issue 19 of CSIMQ closes with the article "A Methodology for Operationalizing Enterprise IT Architecture and Evaluating its Modifiability" that discusses robust methods with which to operationalize enterprise IT architecture, in a way enabling to analyze performance, in terms of the ability to adapt and evolve over time. The study proposes a methodology for analyzing enterprise IT architecture based on Design Structure Matrices (DSMs), which capture the coupling between all components in the architecture by capturing the architecture "in-use", structuring it, and generating measures that can be used to analyze performance. The methodology is illustrated with the case and data of a large pharmaceutical firm, showing that the coupling derived from an IT architecture and DSM can predict IT modifiability.

For this thematic issue we owe thanks to the members of CSIMQ's Editorial Review Board for providing valuable reviews for the submitted articles. Special thanks go to the Managing Editor of the CSIMQ - Prof. Marite Kirikova for her great support in realizing this thematic issue, as well as to the publicizing team of the CSIMQ journal for their professional help and efficiency. Finally, we are grateful to the authors for submitting the articles reporting their scientifically innovate and passionate work.

To all readers - we hope you will enjoy this issue and use it to conceive novel ideas in the actual topics of Business Informatics. 